\title{
Effect of Dioscorea Pills on Immunity of Mice Receiving Chemotherapy for Ovarian Cancer
}

\author{
Yiming Tang1, Huijun $\mathrm{Xu}^{2}$ \\ ${ }^{1}$ Institute of Integrative Medicine, Qingdao University Medical College, Qingdao, China \\ ${ }^{2}$ Qingdao Haici Hospital, Qingdao, China \\ Email: $241955448 @ q q . c o m$
}

How to cite this paper: Tang, Y.M. and $\mathrm{Xu}$, H.J. (2019) Effect of Dioscorea Pills on Immunity of Mice Receiving Chemotherapy for Ovarian Cancer. Open Access Library Journal, 6: e5247.

https://doi.org/10.4236/oalib.1105247

Received: February 14, 2019

Accepted: February 25, 2019

Published: February 28, 2019

Copyright () 2019 by author(s) and Open Access Library Inc.

This work is licensed under the Creative Commons Attribution International License (CC BY 4.0).

http://creativecommons.org/licenses/by/4.0/

(c) () Open Access

\begin{abstract}
Objective: To observe the effect of Dioscorea pills on the immunity of mice with ovarian cancer after chemotherapy and its mechanism of action. Methods: $\mathrm{BALB} / \mathrm{C}$ mice were randomly divided into blank group, ovarian cancer model group, chemotherapy group and chemotherapy plus TCM (Traditional Chinese Medicine) group with 20 mice in each group. Except the blank group, 60 mice in other groups were subcutaneously injected with SKOV-3 ovarian cancer cell suspension at the right buttock to build a tumor-bearing mouse model. After tumor formation, the chemotherapy group and the chemotherapy plus TCM group were intraperitoneally injected with Docetaxel. Next day, the chemotherapy plus TCM group was given Dioscorea pills by gavage, while the other three groups were given equal quantity of normal saline. All mice were sacrificed 21 days after treatment. The white blood cell count and serum levels of IL- 2 and TNF- $\alpha$ were measured. Results: Compared with the model group and the ovarian cancer model group, the levels of IL-2 and white blood cell count of the chemotherapy group and the chemotherapy plus TCM group were significantly decreased $(\mathrm{P}<0.05)$, while the levels of TNF- $\alpha$ were significantly increased $(\mathrm{P}<0.05)$. Compared with the chemotherapy group, the levels of IL-2 of the chemotherapy plus TCM group were greatly increased $(\mathrm{P}<0.05)$; the level of TNF- $\alpha$ was slightly increased without significant difference $(\mathrm{P}>0.05)$. Conclusions: Dioscorea pills can improve the immunity of mice with ovarian cancer after chemotherapy.
\end{abstract}

\section{Subject Areas}

Immunology

\section{Keywords}

Dioscorea Pill, Mouse with Ovarian Cancer, Whitebloodcell, IL-2, TNF- $\alpha$ 


\section{Introduction}

Ovarian cancer is one of the most common malignant tumors of female reproductive organs, and the incidence rate is high [1]. It is ranked third after cervical cancer and endometrial cancer. However, ovarian cancer is the most common gynecological malignant tumor with a five-year survival rate of only $20 \%$ to $30 \%$ [2]. At present, the treatment of ovarian cancer is mainly based on the comprehensive treatment of surgical treatment plus radiotherapy and chemotherapy. Chemotherapy is an important means of treating ovarian cancer. However, with the deepening of treatment, the toxic side effects of chemotherapeutic drugs have become increasingly prominent [3]. The killing effect of chemotherapeutic drugs on normal cells, especially highly sensitized lymphocytes, often leads to a significant decrease in immune function. According to the clinical symptoms and signs of ovarian cancer, it is the disease category of the motherland medicine "sickness", “intestinal fistula", “accumulation", "swelling”. A large number of clinical studies have shown that Chinese medicine has a definite therapeutic effect in improving the body's immune function. Traditional Chinese medicine, with its unique drug and drug characteristics, plays an important role in the treatment of ovarian cancer, especially in reducing the side effects of chemotherapy drugs and enhancing the immune function of chemotherapy individuals.

In this study, by copying the $\mathrm{BALB} / \mathrm{C}$ mouse ovarian cancer model, a comparative study of chemotherapy and chemotherapy + yam pills intervention was used to detect the number of white blood cells, interleukin-2 (IL-2) and tumor necrosis in the blood of mice. The content of factor $\alpha$ (TNF- $\alpha$ ) demonstrates and explores that the intervention of Dioscorea opposita can improve the immune function and its mechanism of ovarian cancer chemotherapy mice.

\section{Material Method}

\subsection{Experimental Materials}

\subsubsection{Experimental Animals}

SPF female BALB/C mice, 100 females, 4 week-old females, weighing about $20 \pm$ $1 \mathrm{~g}$, were provided by the Experimental Animal Center of Qingdao University, and standardized feeding in the SPF animal room of the clinical experiment center. All feeding, manipulation and experimental protocols for experimental mice were performed in accordance with the standard animal welfare guidelines and were approved by the ethics committee of our school.

\subsubsection{Experimental Cells}

Human ovarian cancer cell SKOV-3, purchased from the Chinese Academy of Sciences Shanghai Cell Bank.

\subsubsection{Experimental Drugs}

Dioscorea decoction preparation: Fried yam 20 g, Angelica 10 g, Dwarf Lilyturf Tuber $5 \mathrm{~g}$, Red peony $5 \mathrm{~g}$, Fried Atractylodes Rhizome $5 \mathrm{~g}$, Almond $5 \mathrm{~g}$, Donkey-hide gelatin 6 g, Cassia Twig 6 g, Rehmannia 10 g, Medicated leaven 10 g, 
Ligusticumwallichii $6 \mathrm{~g}$, Bupleurum $6 \mathrm{~g}$, Campanulaceae $5 \mathrm{~g}$, Poriacocos $5 \mathrm{~g}, \mathrm{Li}$ corice 18 g, Codonopsis 5 g, Dried ginger 3 g, Radix ampelopsis 3 g, Divaricate Saposhniovia Root 5 g, Fried Coix Seed 6 g, Jujube 30 g. All Chinese herbal medicines were purchased from the Chinese Pharmacy of Qingdao Haici Medical Group. According to the "the table of body surface area conversion equivalent dose ratio" to convert the dose of mice with yam glutinous rice balls, the yam recipe pills are fried for 5 days once. Decoction method: $300 \mathrm{ml}$ water is diluted with raw medicine for half an hour, boiled with artificial intelligent electric decocting casserole, and then the liquid is evaporated and concentrated to $12 \mathrm{ml}$, the concentration of crude drug is $1.5 \mathrm{~g} / \mathrm{ml}$, and the antibacterial concentration is stored for use in the environment of $4^{\circ} \mathrm{C}$ after sealing. The same method is used to prepare a plurality of yam pills. Chemotherapeutic drugs: Docetaxel (Jiangsu Pfizer Pharmaceutical Co., Ltd.) was purchased from the western pharmacy of Qingdao Haici Medical Group.

\subsubsection{Experimental Instruments and Reagents}

The automatic blood cell counter, fetal bovine serum and RPM I1640 medium were purchased from GIBCO, USA, and IL-2 and TNF- $\alpha$ kits were purchased from Beijing Chaoyan Biological Co., Ltd.

\subsection{Experimental Methods}

\subsubsection{Grouping of Test Subjects}

100 SPF female BALB/C mice were randomly divided into blank control group, ovarian cancer model group, chemotherapy alone group and chemotherapy + yam pills intervention group, 25 rats in each group.

\subsubsection{Cell Culture}

Standardized subculture of human ovarian cancer cell line SKOV-3, 10\% fetal bovine serum RPM 1640 medium, $5 \% \mathrm{CO}_{2}$ saturated humidity in a $37^{\circ} \mathrm{C}$ constant temperature incubator standardized non-polluting culture to cell coverage rate of $90 \%$ to $95 \%$ for cell passage.

\subsubsection{Reproduction of Ovarian Cancer Mouse Model}

Human ovarian cancer cell line SKOV-3 in logarithmic growth phase was washed and PBS buffered and diluted to adjust the concentration. The cell concentration was determined to be $1 * 10^{\wedge} 7 / \mathrm{ml}$ by spectrophotometer. Ovarian cancer model group and chemotherapy alone combination All mice in the ovarian cancer + dioscorea interfering group were subcutaneously inoculated with $0.1 \mathrm{ml}$ cell suspension on the right side of the hip. The operation standard was observed during the inoculation, and infection prevention was prevented to prevent the mice from accidentally dying due to the injection operation. All mice in the blank control group were injected with the same amount of normal saline at the same site. The standardized feeding was continued for 1 week, and the chemotherapy experimental procedure was prepared when a $0.5 \mathrm{~cm} * 0.5 \mathrm{~cm}$ tumor mass was formed. 


\subsubsection{Implementation of Chemotherapy and Yam Pill Interference}

The subcutaneous tumor mass was formed on the 8th day, and all the mice in the chemotherapy group, chemotherapy + yam pills intervention group were treated with docetaxel (docetaxel injection with sterile saline to $0.1 \mathrm{ml}$ ). One injection was given, and the docetaxel dose standard was $6.28 \mathrm{mg} / \mathrm{kg}$. The blank control group and the ovarian cancer model group were injected subcutaneously with $0.1 \mathrm{ml}$ of normal saline right hip. Two days after the completion of the injection operation, the chemotherapy alone combined chemotherapy + diosgenin interference group received daily oral administration of $0.3 \mathrm{ml}$ of diosgenin decoction at 8 o'clock in the morning, and the other three groups were treated with $0.3 \mathrm{ml}$ of normal saline. The drug was administered three weeks later.

\subsubsection{Specimens Taken}

During the whole experiment, 1 mouse in the ovarian cancer model group died without abnormal symptoms, and 1 mouse in the chemotherapy alone group died improperly. The chemotherapy + yam pills interfered with 2 mice and died due to improper operation. All mice were fasted one night in advance. The next morning, each group of surviving mice was randomly divided into 20 groups of 4 mice, and each group of mice was treated with eye venous blood. And do all the markings, make a mark blood cell count sample (full blood cell counter test), and make a labeled serum sample, the production method (fixed mice, lift the eyeball skin, fully expose the eyeball, use the eyeball scissors to quickly cut off the eyeball The blood was dropped into the labeled centrifuge tube, and each mouse was allowed to stand for $20 \mathrm{~min}$, centrifuged $\left(3000 \mathrm{r} / \mathrm{min} 4^{\circ} \mathrm{C}\right.$ for 10 $\mathrm{min}$ ), the upper serum was taken, and the spare mice were sacrificed by cervical dislocation.

\subsubsection{Indicator Detection}

All the blood cell count samples were sent to the test center for white blood cell metering and recording by whole blood cell counter, and the expression levels of IL- 2 and TNF- $\alpha$ in each group were detected by ELISA. All samples were labeled with serum, washed with enzyme plate, and blank control wells and sample detection wells were added. Each well was tested with $50 \mathrm{ul}$ of test sample (sample to be tested:sample dilution $=1: 4$ ), sealing plate was sealed, and thermostat was kept at $37^{\circ} \mathrm{C}$. Incubate for half an hour, wash the plate, except for the blank control well, add $50 \mathrm{ul}$ of enzyme labeling reagent to each well, seal the plate, incubate at $37^{\circ} \mathrm{C}$ for half an hour, wash the plate, add $50 \mathrm{ul}$ of $\mathrm{A}$ and $\mathrm{B}$ coloring agent in each well, fully shake and mix. At $37^{\circ} \mathrm{C}$, the temperature was kept at a constant temperature for $15 \mathrm{~min}$, and $50 \mathrm{ul}$ of the stop solution was added to stop the reaction. The absorbance was measured at a wavelength of $450 \mathrm{~nm}$ by a microplate reader (recording OD value). The concentration values of IL-2 and TNF- $\alpha$ in each sample were calculated according to the standard curve.

\subsection{Statistical Methods}

Statistical data were processed by SPSS.23.0 statistical software. The data were 
expressed as mean \pm standard deviation $(\mathrm{x} \pm \mathrm{s})$. One-way ANOVA was used to compare and analyze all the measured data. The comparison between groups was performed by LSD-t test. $\mathrm{P}<0.05$ was considered statistically significant.

\section{Results}

\subsection{The Test Results of White blood Cell Count}

There were significant differences between the chemotherapy group and the blank control group and the ovarian cancer model group $(\mathrm{P}<0.05)$, which proved that the ovarian cancer model was successfully replicated and met the experimental conditions. There was significant difference between the ovarian cancer model group and the chemotherapy alone group $(\mathrm{P}<0.05)$, which proved that the chemotherapy drugs had obvious damage and inhibition on the immune function of ovarian cancer mice. There was also a statistically significant difference between the chemotherapy + yam pills intervention group and the ovarian cancer model group and the chemotherapy alone group $(\mathrm{P}<0.05)$. It was confirmed that the intervention of diosgenin pills can increase the number of white blood cells in ovarian cancer chemotherapy mice and improve their immunity (Table 1).

\subsection{Tumor Tissue Staining}

Randomly selected mice from ovarian cancer model group, chemotherapy alone group, chemotherapy + yam pills intervention group were used to observe part of the tumor tissue for microscopic observation and immunofluorescence staining. The picture is as follows. As can be seen from Figures 1-6, the tumor cells are concentrated in a patchy form, with large nuclei, large nuclear-to-nuclear ratio, deep staining of nucleoli, low degree of differentiation, active division, and more necrotic tissue in the center of the tumor tissue. According to histological judgment, the cancer tissue was successfully replicated.

Observed under the Opticalmicro scope: Representative micrographs of cells collected from the three groups. The amplification time is $10 * 10$.

Immunofluorescence: Fluorescence imaging amplification time is $40 * 10$. Green represents the expression of ovarian cancer cell line LGR5.

Table 1. The test results of white blood cell of four groups of mice $\left( \pm \mathrm{s}^{\star} 10^{\wedge} 3 / \mathrm{mm}^{3}\right)$.

\begin{tabular}{ccc}
\hline Group & Number of samples tested & White blood cell count \\
\hline Blank control group & 20 & $9.26 \pm 0.52$ \\
Ovarian cancer model group & 20 & $9.37 \pm 0.61$ \\
Chemotherapy alone & 20 & $5.04 \pm 0.22$ \\
Chemotherapy + Dioscorea Pill & & $7.85 \pm 0.39$ \\
\hline Interference Group & 20 & \\
\hline
\end{tabular}

Note: $\mathrm{P}=0.000<0.05$ compared with the blank control group in the chemotherapy alone group. Compared with the ovarian cancer model group, the chemotherapy alone group had $\mathrm{P}=0.000<0.05$. The chemotherapy + yam pills intervention group compared with the ovarian cancer model group, $\mathrm{P}=0.000<0.05$. Chemotherapy + Dioscorea in the intervention group compared with the chemotherapy alone group, $\mathrm{P}=0.000<0.05$. 


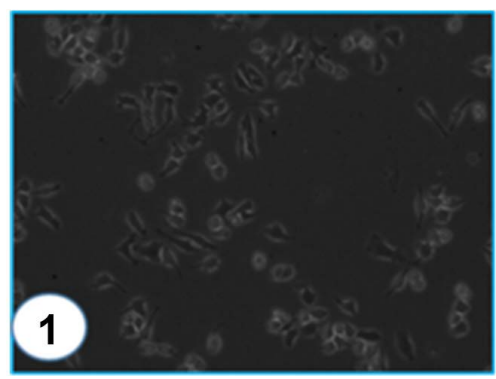

Figure 1. Ovarian cancer model group.

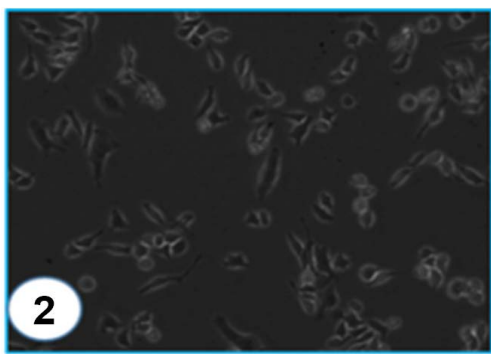

Figure 2. Chemotherapy alone.

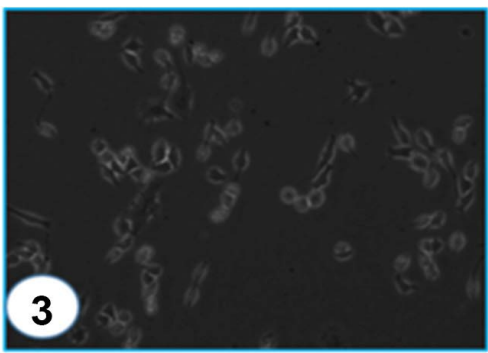

Figure 3. Chemotherapy + Dioscorea Pill Interference Group.

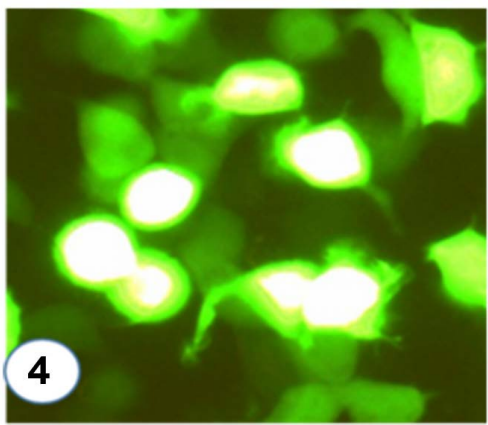

Figure 4. Ovarian cancer model group.

\subsection{Expression of IL-2, TNF- $\alpha$ and IFN- $\gamma$}

As shown in Table 2: Compared with the blank control group, the IL-2 content of the ovarian cancer model group decreased, and there was a statistical difference $(P=0.016)$. Compared with the ovarian cancer model group, the TNF- $\alpha$ content decreased, and there was a statistical difference $(P=0.000)$. 


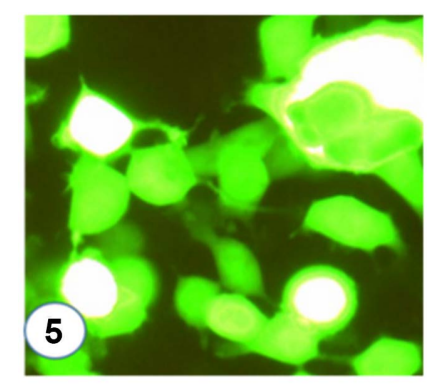

Figure 5. chemotherapy alone.

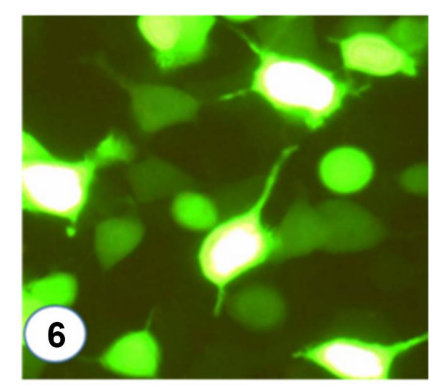

Figure 6. Chemotherapy + Dioscorea Pill Interference Group.

Table 2. Expression of IL-2 and TN F-a in serum of each group (pg/ml, \pm s).

\begin{tabular}{cccc}
\hline Group & Number of samples & IL-2 & TNF- $\alpha$ \\
\hline Blank control group & 20 & $30.01 \pm 1.09$ & $586.48 \pm 114.01$ \\
Ovarian cancer model group & 20 & $28.99 \pm 1.61$ & $347.17 \pm 100.12$ \\
Chemotherapy alone & 20 & $23.24 \pm 1.50$ & $540.01 \pm 111.23$ \\
$\begin{array}{c}\text { Chemotherapy + Dioscorea Pill } \\
\text { Interference Group }\end{array}$ & 20 & $26.94 \pm 1.49$ & $575.31 \pm 89.49$ \\
P value & & 0.000 & 0.001 \\
F value & & 30.996 & 8.457 \\
\hline
\end{tabular}

Compared with the ovarian cancer model group, the IL-2 content in the chemotherapy group was significantly lower $(P=0.000)$, and the IL-2 content in the chemotherapy + yam pills intervention group was statistically different $(\mathrm{P}=$ 0.002).

Compared with the ovarian cancer model group, the TNF- $\alpha$ levels in the chemotherapy group and the chemotherapy + yam pills intervention group were significantly higher $(\mathrm{P}=0.002$ and 0.001$)$.

Compared with the chemotherapy alone group, the IL- 2 content in the chemotherapy + yam pills intervention group increased, the difference was statistically significant $(\mathrm{P}=0.012)$, but the TNF- $\alpha$ content increased to some extent, but the difference was not statistical. Meaning $(\mathrm{P}=0.571)$.

According to the statistical analysis of results, it can be explained that BALB/C mice with ovarian cancer have impaired immune function, and chemotherapy 
treatment further impairs the immune function of mice. After the intervention treatment with the application of diosgenin, the immune function of the mice was improved and improved to some extent. The rising trend of TNF-a may be caused by the interaction between docetaxel and tumor necrosis factor, which needs further exploration.

\section{Discussion}

Ovarian cancer is a malignant tumor that occurs in the epithelial tissues of the ovary. It is one of the major diseases that threaten the health of women. Modern medicine believes that abnormal immune function is an important cause of tumor development. For tumor tissue, immunity, especially cellular immunity, is the most important immune pathway against tumors. In the process of cellular immunity, IL- 2 and TNF- $\alpha$ produced by T cells, especially TH1 cells, are one of the most important cytokines involved in immunity [4]. Studies have shown that IL-2 is one of the most important mediators of activation-mediated immune cells such as macrophages and NK cells to kill tumor cells [5]. TNF- $\alpha$ has a direct effect on directly killing tumor cells, inhibiting tumor activity, and preventing tumor metastasis. TNF-a can directly act on the DNA of tumor cells, leading to cell disintegration or atrophy. Therefore, the increase of IL-2 and TNF- $\alpha$ contributes to the body's clearance and anti-tumor, and is an important indicator of the body's immune function [6].

Modern medical treatment of ovarian cancer is mostly surgery + radiotherapy and chemotherapy. Early radical resection of the tumor and adjuvant chemotherapy and radiotherapy have a good effect on the radical treatment of ovarian cancer, but the side effects caused by chemotherapy drugs are often not negligible. Especially at the end of the chemotherapy course, the treatment method of "killing one thousand and self-losing eight hundred" greatly impaired the immune function of the patient and affected the physical and mental health of the patient. In the late stage of chemotherapy, the blood test results of patients often appear as "low white blood image" [7]. The number of white blood cells is often one of the important indicators to measure whether patients can continue to complete chemotherapy. Many patients have to suspend the original chemotherapy cycle because of "low white blood image". The combination of the recipes of the yam and the medicinal herbs is ingenious, and the intention of using the medicine is to supplement the remedy. Modern pharmacological studies have shown that the aglycone of Dioscoreazingi berensis is diosgenin, which has anti-tumor and immune-enhancing effects [8]. Patients with postoperative ovarian cancer and after radiotherapy and chemotherapy often have typical debilitating states such as body weight loss, fatigue, dizziness, facial dysfunction, anemia, shortness of breath, loss of appetite, sore throat, loose stools, and repeated colds. In clinical work, the application of diosgenin pills to treat diseases often requires identification with Zhigancao Decoction and Chaiqin Decoction [9]. In addition, Dioscorea granules should be used for the treatment of patients with malignant 
tumors such as ovarian cancer and patients undergoing radiotherapy and chemotherapy [10]. Because the prescription is relatively long, the patients should be relieved when they apply for clinical use. The merits of the day, in the long-term benefits of the map, the stomach rises and then the Gubangan, this solid and evil cannot invade Yuan Pei and the disease is self-destructive [11]. Therefore, Dioscorearum is expected to become an important application in the field of ovarian cancer treatment, and has important application value for patients with ovarian cancer after surgery and chemoradiotherapy. In recent years, the prescription has been increasingly used in other clinical diseases, and has achieved good results in the fields of tuberculosis, peptic ulcer, postpartum low back pain, urticaria and chemotherapy adjuvant therapy in patients with cancer [12]. At the same time, through the continuous exploration and application of medical colleagues, the prescription has also achieved many beneficial practices in the fields of cardiovascular and cerebrovascular diseases and autoimmune diseases, creating a healthy opportunity for more and more patients [13].

In this study, we replicated the ovarian cancer BALB/C mouse model and used it as a research object to investigate the effect of Dioscorea on the immune function of ovarian cancer chemotherapy mice. According to the experimental results, it is concluded that the interference of Dioscorea zingiberensis can definitely improve the autoimmune power of ovarian cancer mice in the chemotherapy stage, that is, reduce the side effects caused by chemotherapy drugs. The main manifestation of improving immunity is to reduce the damage of chemotherapy drugs to white blood cells, to Increases the levels of IL- 2 and TNF- $\alpha$ and support tumor clearance and toxic side effects of chemotherapy drugs. Although the experimental subjects are female $\mathrm{BALB} / \mathrm{C}$ mice, according to the experimental results, it still has reference significance for clinical practice [14]. Moreover, according to the prescription ratio of the yam recipe, combining the important exploration and practice of ancient and modern doctors, combined with the field research results of this study, the following conclusions can be drawn: the intervention of yam pills can improve the immune function of ovarian cancer chemotherapy mice. It has reference significance for clinical treatment of ovarian cancer. The dialectical application of Dioscorea pills has important theoretical and practical value for clinical treatment of patients with ovarian cancer. Dioscorearum is expected to become an important prescription and treatment for ovarian cancer treatment, and its clinical efficacy needs to be further explored and supplemented by the majority of medical colleagues.

\section{Conflicts of Interest}

The authors declare no conflicts of interest regarding the publication of this paper.

\section{References}

[1] Jemal, A., Bray, F., Center, M.M., et al. (2015) Global Cancer Statistics. Ca A Cancer Journal for Clinicians, 65, 87-108. 
[2] Tang, Z.Y. (2011) Modern Oncology.

[3] Yang, B.J., Li, S. and Chen, R. (2014) New Progress in the Treatment of Ovarian Cancer with Traditional Chinese Medicine. Journal of Liaoning University of Traditional Chinese Medicine, No. 5, 121-124.

[4] Thakur, A., Schalk, D., Sarkar, S.H., et al. (2012) A Th1 Cytokine-Enriched Microenvironment Enhances Tumor Killing by Activated T Cells Armed with Bispecific Antibodies and Inhibits the Development of Myeloid-Derived Suppressor Cells. Cancer Immunology Immunotherapy, 61, 497-509. https://doi.org/10.1007/s00262-011-1116-1

[5] Chandler, S.W., Rassekh, C.H., Rodman, S.M., et al. (2002) Immunohistochemical Localization of Interleukin-10 in Human Oral and Pharyngeal Carcinomas. Laryngoscope, 112, 808-815. https://doi.org/10.1097/00005537-200205000-00008

[6] Li, Y., Liang, W., Liu, W.B., et al. (2004) Effects of Chemotherapy on Th1 and Th2 Cytokine Drift in Gastric Cancer and Its Clinical Significance. Chinese Journal of Oncology, 26, 732-734.

[7] An, L.J. (2013) Current Status and Progress of Diagnosis and Treatment of Ovarian Cancer. Chinese and Foreign Medical Research, No. 19, 147-149.

[8] Zhang, Z.J. (1993) Jin Gui's Theory of Slightly. China Bookstore, Beijing.

[9] Ouyang, Q. (1987) The Original Purpose of Miscellaneous Diseases. People's Medical Publishing House, Beijing.

[10] Yu, H.Q. and Yu, J.S. (2013) Understanding of the "Golden Dragonfly" DioscoreaPills. Chinese Journal of Basic Medicine in Traditional Chinese Medicine, No. 7, 817-818.

[11] Zhao, D.Z., Xie, S., Zhang, Y., et al. (2016) Discussion on the Mechanism of Yam Pill Reuse of Yam. Jilin Journal of Traditional Chinese Medicine, No. 4, 398-401.

[12] Feng, S. and Li, H.M. (2012) Overview of Clinical Application and Research of Dioscoreapills. Zhejiang Journal of Traditional Chinese Medicine, 47, 699-701.

[13] Miao, T.T. (2016) Huang Huang's Clinical Experience in Treating Lung Cancer after Chemotherapy with Dioscorea Pill. Journal of Nanjing University of Traditional Chinese Medicine, No. 2, 198-200.

[14] Lin, J.X., Xie, S., Li, L.H., et al. (2015) Clinical Study on the Effect of Dioscorea on the Attenuation and Synergism of Breast Cancer Patients during Chemotherapy. World Journal of Traditional Chinese Medicine, No. 10, 1540-1542. 